\title{
The immune response to sand fly salivary proteins and its influence on Leishmania immunity
}

\section{Regis Gomes and Fabiano Oliveira*}

Laboratory of Malaria and Vector Research, National Institutes of Health, National Institute of Allergy and Infectious Disease, Rockville, MD, USA

\section{Edited by:}

Nathan Peters, National Institute of

Allergy and Infectious Diseases, USA

\section{Reviewed by:}

David M. Mosser, University of Maryland, USA

Matthew Edward Rogers, London

School of Hygiene and Tropical

Medicine, UK

\section{*Correspondence:}

Fabiano Oliveira, Vector Molecular

Biology Section, Laboratory of

Malaria and Vector Research, National

Institutes of Health, National Institute of Allergy and Infectious Disease,

12735 Twinbrook Parkway, 2E-22b,

Rockville, MD 20852, USA.

e-mail: loliveira@niaid.nih.gov
Leishmaniasis is a vector-borne disease transmitted by bites of phlebotomine sand flies. During Leishmania transmission, sand fly saliva is co-inoculated with parasites into the skin of the mammalian host. Sand fly saliva consists of roughly thirty different salivary proteins, many with known roles linked to blood feeding facilitation. Apart from the anti-hemostatic capacity of saliva, several sand fly salivary proteins have been shown to be immunogenic. Immunization with a single salivary protein or exposure to uninfected bites was shown to result in a protective immune response against leishmaniasis. Antibodies to saliva were not required for this protection. A strong body of evidence points to the role for salivaspecific T cells producing IFN- $\gamma$ in the form of a delayed-type hypersensitivity reaction at the bite site as the main protective response. Herein, we review the immunity to sand fly salivary proteins in the context of its vector-parasite-host combinations and their vaccine potential, as well as some recent advances to shed light on the mechanism of how an immune response to sand fly saliva protects against leishmaniasis.

\footnotetext{
Keywords: sand fly, salivary proteins, Leishmania, Lutzomyia longipalpis, Phlebotomus papatasi, Phlebotomus duboscqi
}

\section{INTRODUCTION}

Leishmaniasis is a vector-borne neglected disease caused by protozoan parasites of the genus Leishmania. The disease is endemic in 98 countries with an estimated 350 million people at risk of acquiring leishmaniasis, an incidence of two million new cases each year, and an estimated burden of 2,357,000 disability-adjusted life years (WHO, 2010). The clinical manifestation includes cutaneous, mucocutaneous, diffuse cutaneous, and visceral leishmaniasis (VL) forms. VL is fatal if untreated and the morbidity and stigma caused by cutaneous leishmaniasis (CL) is considerable (Desjeux, 2004).

Leishmaniasis is transmitted to the host by an infected sand fly bite. During feeding, the female phlebotomine will probe the skin lacerating superficial capillaries and leading to the formation of a blood pool. Sand flies will actively secrete salivary proteins into the dermis in order to facilitate blood feeding, avoiding hemostasis, and reducing inflammation (Lerner et al., 1991; Charlab et al., 1999; Kamhawi, 2000). The significance of sand fly saliva in leishmaniasis research was captured some 25 years ago (Ribeiro, 1987) with the demonstration that sand flies are much more than just chauffeurs for Leishmania parasites. The fact that sand fly salivary proteins are obligatorily inoculated with parasites within the skin of the vertebrate host is an important initial step that is still overlooked, particularly because protective vaccines against Leishmania infective-challenge delivered by needle failed to control a vector-borne challenge (Peters et al., 2009) while multiple exposures to sand fly saliva induce an immune response that can control leishmaniasis in animal models (Belkaid et al., 1998). Transcriptomics of sand fly salivary glands led to the identification of the secreted salivary proteins and the characterization of the function and immunogenic potential for some of these proteins (Oliveira et al., 2009). Salivary proteins with protective immune responses against leishmaniasis in animal models were identified but challenges remain to validate their protective role in humans. This review will focus on the alterations of the host immune system by anti-saliva immunity and how in turn it can control leishmaniasis.

\section{SAND FLY SALIVA ENHANCES LEISHMANIA INFECTION}

The classical work by Titus and Ribeiro (1988) demonstrated that the infection with Leishmania major is significantly exacerbated by the presence of Lutzomyia longipalpis saliva, a New World sand fly. Its saliva contains a $6.5-\mathrm{kD}$ peptide named maxadilan, which is a well-characterized salivary molecule working as a potent vasodilator (Lerner et al., 1991). Maxadilan can also act by inhibiting or modulating the inflammatory and immune response of mice, suggesting it contributes to the disease-exacerbating qualities of $L$. longipalpis saliva (Morris et al., 2001). The addition of maxadilan to mouse macrophage upregulates cytokines associated with Th2 response (IL-6, IL-10, and TGF- $\beta$ ) but downregulates Th1 cytokines (IL-12p70 and TNF) and nitric oxide (NO) (Brodie et al., 2007). Dendritic cells (DC) exposed to maxadilan showed reduced expression of co-stimulatory molecules (CD80 and CD86) and chemokine (CCR7) expression and induced secretion of type 2 cytokines suggesting that maxadilan can act not only on DC phenotype, but also on the function of those cells, which can be important to control the infection against Leishmania (Wheat et al., 2008). Maxadilan also inhibits $\mathrm{T}$ cell activation, decreasing the production of TNF, $\mathrm{H}_{2} \mathrm{O}_{2}$, and nitric oxide (NO), molecules that are important in the destruction of L. major (Qureshi et al., 1996; Soares et al., 1998; Gillespie et al., 2000). It was recently shown that $L$. longipalpis saliva induced lipid body formation in murine macrophages in vitro and in vivo and these lipid bodies were linked 
with the production of prostaglandin E2 (Araujo-Santos et al., 2010) a molecule that could act on parasite dissemination. Consistently with previous results using murine macrophages or monocytes, the incubation of $P$. papatasi, P. sergenti, or L. longipalpis saliva reduced proliferation of mitogen-activated murine splenocytes and markedly inhibited the production of the Th1 cytokine IFN- $\gamma$ (Rohousova et al., 2005b). Human DC, neutrophils, and monocytes were also affected by L. longipalpis saliva incubation. L. longipalpis saliva induced apoptosis of neutrophils leading to an increase in parasite burden (Prates et al., 2011), altered the expression of co-stimulatory molecules in DC, macrophages, and monocytes (Costa et al., 2004) and down-regulated the production of TNF and IL-12p40 in LPS-stimulated monocytes (Costa et al., 2004). Trying to mimic the natural route of infection in a murine model of CL, Belkaid et al. (1998) demonstrated that the injection of a low number of $L$. major plus saliva of its natural vector Phlebotomus papatasi also enhanced the infection in the ear dermis of naive mice. AMP and adenosine present in P. papatasi saliva seems to be involved in the exacerbation observed in this model (Ribeiro et al., 1999; Katz et al., 2000). Adenosine is an immunomodulatory component, inducing IL-10 production and suppressing TNF- $\alpha$ and IL-12 in the mouse model (Hasko et al., 1996, 2000). It also has the capacity to down-regulate the expression of the inducible nitric oxide (NO) synthase gene in activated macrophage and inhibits the production of $\mathrm{NO}$ as well (Katz et al., 2000). AMP and adenosine from $P$. papatasi saliva were used to treat an experimental murine model of arthritis. These molecules acted on DC function reducing the Th17 immune response and suppressing the autoimmune response (Carregaro et al., 2011). Additionally, it was demonstrated that $P$. papatasi saliva induces IL-4 at the site of inoculation in mice and that effect was abrogated when IL-4 knockout mice were used (Belkaid et al., 1998). This body of work highlights the Th2 potential of sand fly saliva helping to explain the consistent exacerbatory role of saliva in leishmaniasis. Nevertheless, treatment of human macrophages with $L$. intermedia saliva resulted in a decrease of IL-10 production and increase in co-stimulatory molecule expression (Menezes et al., 2008). Interestingly, if L. braziliensis is present during the treatment of macrophages with saliva, it leads to an increase in production of TNF, IL-6, and IL- 8 pro-inflammatory cytokines without a modification in the parasite loads (Menezes et al., 2008). Differences in the composition of the saliva of these vectors may explain the conflicting roles of saliva after in vitro incubation.

\section{IMMUNITY TO SAND FLY BITES}

Sand fly saliva has immunomodulatory properties, but it is important to point out that many of these components are proteins and therefore have the potential to be immunogenic. In fact it has been shown that multiple exposures to sand fly bites induce specific immunity that can be detected by the presence of antibodies and cellular immune responses. It is remarkable that all tested vector sand fly species from New and Old World were able to induce immune responses in several hosts. In laboratory settings, immune responses to the sand fly salivary proteins have been consistently shown in mice, hamsters, dogs, and humans following repeated exposure to bites or by injection of salivary glands dissected from female $P$. argentipes, $P$. ariasi, $P$. papatasi, P. sergenti, L. longipalpis, and L. intermedia (Ghosh and Mukhopadhyay, 1998; Belkaid et al., 2000; Kamhawi et al., 2000; Morris et al., 2001; Valenzuela et al., 2001; Silva et al., 2005; Thiakaki et al., 2005; Oliveira et al., 2006, 2008; de Moura et al., 2007; Jochim et al., 2008; Collin et al., 2009; Drahota et al., 2009; Ahmed et al., 2010; Ben Hadj Ahmed et al., 2010; Clements et al., 2010; Rohousova et al., 2011; Vlkova et al., 2011). Additionally, hosts living in sand fly prevalent areas also present with an immune response to sand fly salivary proteins. The presence of antibodies in humans, dogs and foxes in endemic areas of leishmaniasis has been well documented raising an opportunity to use anti-saliva antibodies as markers of vector exposure (Barral et al., 2000; Gomes et al., 2002, 2007; Rohousova et al., 2005a; Silva et al., 2005; Bahia et al., 2007; de Moura et al., 2007; Vinhas et al., 2007; Hostomska et al., 2008; Aquino et al., 2010; Souza et al., 2010; Teixeira et al., 2010; Vlkova et al., 2011). Apart from being epidemiological tools, anti-saliva antibodies have been linked to increased risk of CL caused by L. tropica (Rohousova et al., 2005a), L. braziliensis (de Moura et al., 2007), or L. major (Marzouki et al., 2011) in Turkey, Brazil, and Tunisia, respectively. On the other hand, the presence of antibodies to salivary proteins from sand fly vectors of VL correlated with protection in humans (Gomes et al., 2002; Aquino et al., 2010) and more recently in dogs (Vlkova et al., 2011). Taken together, these data suggest that the association of anti-sand fly saliva antibodies and risk of CL or $\mathrm{VL}$, albeit contradictory, are probably not random. We can speculate that neutralization of sand fly salivary proteins by anti-saliva antibodies during the initial events after sand fly transmission of parasites may be linked with protection from VL. Blockage of salivary bioactive molecules that act on hemostasis could impair migration of parasitized cells to the peripheral circulation diminishing Leishmania infection in the liver, bone marrow, and spleen. Nevertheless, a role of immune complexes generated by anti-saliva antibodies could likewise lead to local vasculitis, inflammation, and worsen local inflammation leading to a greater availability of harboring cells in the skin, particularly neutrophils, that could exacerbate the outcome of CL. It is unlikely that the causal explanation is due to an increased preference of the sand fly vectors for some individuals in these endemic areas, leading to a higher selective exposure to sand fly bites. The role of antibodies to sand fly saliva during parasite transmission, establishment, and disease manifestation needs further scrutiny and maybe related to factors pertinent to the VL systemic and CL local pathophysiology.

Protection against CL and VL due to anti-saliva cellular immunity has been well established in rodents (Belkaid et al., 2000; Kamhawi et al., 2000; Morris et al., 2001; Valenzuela et al., 2001; Gomes et al., 2008; Oliveira et al., 2008; Ben Hadj Ahmed et al., 2011; Rohousova et al., 2011; Xu et al., 2011). Table 1 summarizes the outcomes of disease from the sand fly and parasite combinations used to test the anti-sand fly saliva protective potential. The use of Leishmania-infected sand fly vector challenge, the most accepted way to mimic Leishmania transmission in nature, has been scarce in the literature. The use of needle inoculation of salivary gland homogenate (SGH) plus Leishmania parasites has been the most used surrogate. Protection has been reliably accomplished when animals immunized with SGH or exposed to bites are challenged by the SGH of the same vector species plus parasites (Table 1). However, recently it has been shown that hamsters 
Table 1 | Protective potential of sand fly saliva against leishmaniasis.

\begin{tabular}{llllll}
\hline Sand fly & Treatment & Animal model & Challenged with & Protection & Reference \\
\hline P. papatasi & SGH & BALB/C, C57BL/6 & L. major + P. papatasi SGH & Yes & $\begin{array}{l}\text { Belkaid et al. (1998), Oliveira et al. (2008), } \\
\text { Ahmed et al. (2010), Ben Hadj Ahmed et al. } \\
\text { (2010), Ben Hadj Ahmed et al. (2011) }\end{array}$ \\
& & & & Kamhawi et al. (2000) \\
P. papatasi & Bites & BALB/C, C57BL/6 & L. major + infected P. papatasi & Yes & Rohousova et al. (2011) \\
P. duboscqi & Bites & BALB/C & L. major + P. duboscqi SGH & Yes & Gomes et al. (2008) \\
L. longipalpis & SGH & Hamster & L. infantum + L. longipalpis SGH & No & Xu et al. (2011) \\
L. longipalpis & SGH & C57BL/6 & L. major + L. longipalpis SGH & Yes & Thiakaki et al. (2005) \\
L. longipalpis & SGH & BALB/C & L. amazonensis + L. longipalpis SGH & Yes & Thiakaki et al. (2005) \\
L. longipalpis & SGH & BALB/C & L. amazonensis + P. sergenti SGH & No & Thiakaki et al. (2005) \\
L. longipalpis & SGH & BALB/C & L. amazonensis + P. papatasi SGH & No & de Moura et al. (2007) \\
L. intermedia & SGH & BALB/C & L. braziliensis + L. intermedia SGH & No & Tavares et al. (2011) \\
L. longipalpis & SGH & Hamster & L. braziliensis + L. intermedia SGH & Yes & Tavares et al. (2011) \\
L. longipalpis & SGH & Hamster & L. braziliensis + L. longipalpis SGH & Yes &
\end{tabular}

immunized with L. longipalpis SGH are protected against challenge with $L$. braziliensis plus SGH from $L$. intermedia or $L$. longipalpis (Tavares et al., 2011). This data may indicate that these two New World sand flies have salivary proteins that are more conserved and hints to some level of antigenic cross-reactivity between the salivary proteins from these two species.

Despite its conspicuous presence, the literature indicates that anti-sand fly saliva antibodies are not required for protection in rodents (Valenzuela et al., 2001). Protective anti-saliva immunity has been correlated with a delayed-type hypersensitivity (DTH) reaction characterized by cellular recruitment of lymphocytes and macrophages to the site of bite. The environment at the bite site in immunized animals becomes rapidly inhospitable to Leishmania parasites with messenger RNA of Th1 cytokines (IFN- $\gamma$ and IL-12) detected as early as $2 \mathrm{~h}$ after bites (Oliveira et al., 2008) and an increased frequency of cells producing IFN- $\gamma$ and IL-12 detected $6 \mathrm{~h}$ post-bite (Kamhawi et al., 2000). We hypothesize that the fast cellular recruitment and Th1 polarization of the bite site environment will result in a less successful Leishmania establishment in the host and lead to an earlier priming of the immune system toward an anti-Leishmania immunity (Figure 1). The key point in the anti-saliva mediated protection is the close proximity between the salivary proteins and the Leishmania parasites in the moments after the infected sand fly bite, where the anti-saliva DTH response will interfere with the initial establishment of Leishmania in the host's skin. The Leishmania parasites establishment is disturbed as collateral damage from the antisaliva immunity. The microenvironment in the host skin will change considerably from a silent infection in naïve skin to a site highly Th1-polarized with the presence of an active cellular infiltrate.

The data regarding the human cellular response to bites of sand flies is sparse. DTH has been shown at the bite site of naïve individuals sensitized to sand fly bites of P. papatasi (Belkaid et al., 2000) and L. longipalpis (Vinhas et al., 2007). Multiple exposures to L. longipalpis induced an anti-saliva cellular immunity measured by an increased frequency of activated $\left(\mathrm{CD} 25^{+}\right)$peripheral lymphocytes and in vitro production of IFN- $\gamma$ upon stimulation with salivary glands. More recently, Abdeladhim et al. (2011) tested individuals from an endemic area of L. major where P. papatasi is prevalent. Peripheral blood cells were stimulated with salivary glands from $P$. papatasi resulting in proliferation and IL-10 production by $\mathrm{CD}^{+}$lymphocytes. When IL-10 was blocked and $\mathrm{CD}^{+}$cells depleted, $\mathrm{CD} 4^{+}$T cells produced IFN- $\gamma$ after stimulation with $P$. papatasi glands. Interestingly, the saliva of $P$. papatasi contains adenosine, a well-characterized inducer of IL-10 and suppressor of Th1 cytokines such as IL-12 and TNF- $\alpha$ (Hasko et al., 2000). The direct effect of the adenosine from the saliva on in vitro stimulation may overwhelm the acquired immune response to sand fly salivary proteins. Adenosine has been shown to be present in large quantities in P. papatasi and P. argentipes saliva (Ribeiro et al., 1999), but absent from L. longipalpis and P. duboscqi (Kato et al., 2007). This fact may explain the discrepancy between the results of stimulation with $L$. longipalpis and $P$. papatasi salivary glands in humans. Follow-up studies correlating the immune response to sand fly salivary proteins over time to the outcome of leishmaniasis will help to establish the role of the immunity to sand fly salivary proteins in protection, susceptibility, and severity of leishmaniasis.

\section{IMMUNE RESPONSE TO SINGLE SAND FLY SALIVARY PROTEINS}

The natural step after the consistent protection observed against leishmaniasis after exposure to uninfected $P$. papatasi bites (Kamhawi et al., 2000) or immunization with the SGH (Belkaid et al., 1998) was the identification of which salivary proteins were accountable for this immunity. The low complexity of sand fly saliva (around thirty salivary proteins) made it feasible to screen for salivary proteins that induced a DTH-Th1 response to SGH, the hallmark of anti-saliva immunity, from several sand fly species. Identification of the protective proteins was first accomplished in the P. papatasi sand fly. A salivary gland protein from P. papatasi called PpSP15 was injected in mice and provided protective immunity against $L$. major when the parasite was co-inoculated with $P$. papatasi SGH (Valenzuela et al., 2001). After DNA immunization with PpSP15, mice developed a strong DTH in response to P. papatasi bites, comparable with that previously described using sand fly bites to sensitize animals (Kamhawi et al., 2000). Interestingly, 


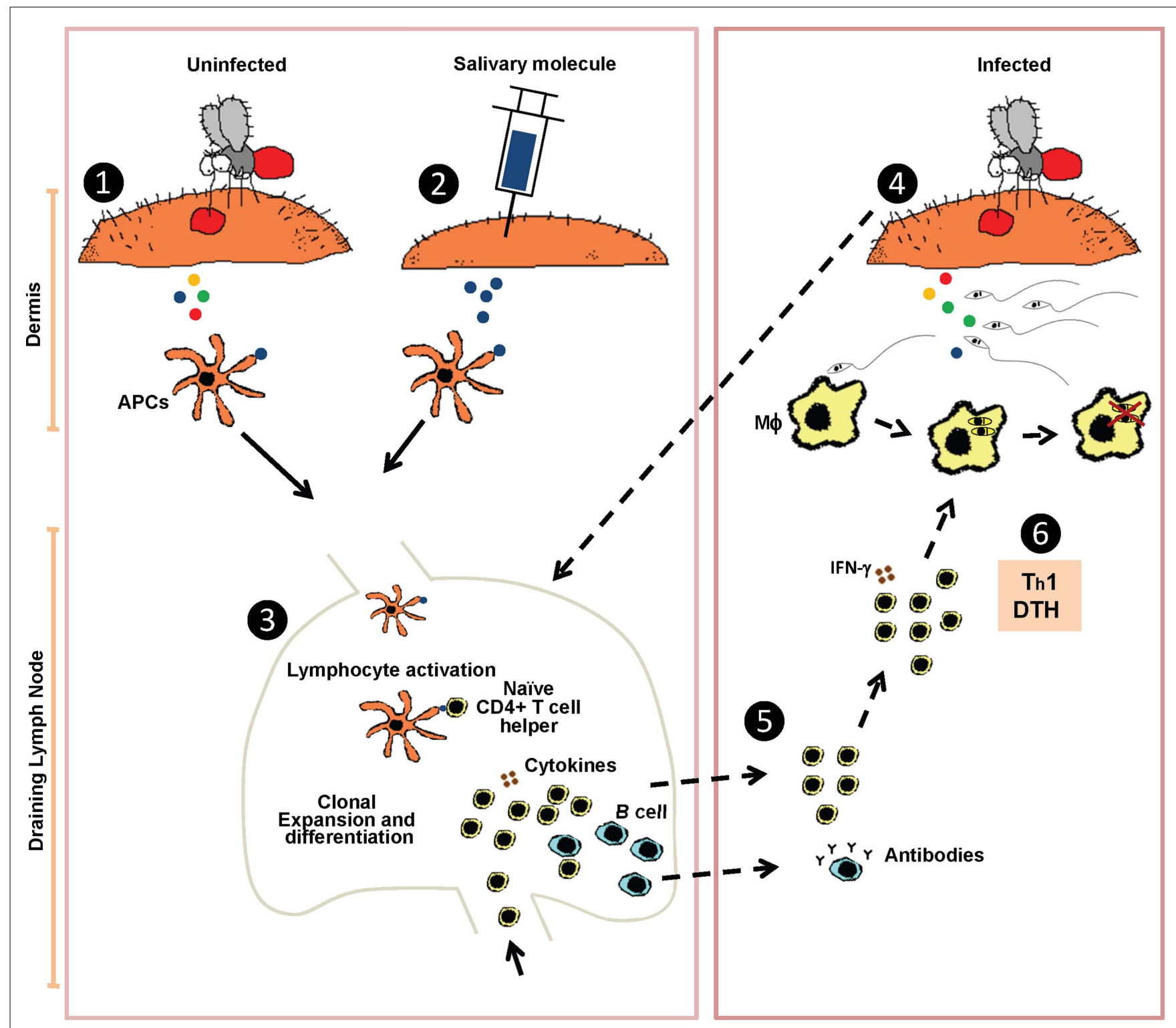

FIGURE 1 | Hypothetical model of the protective mechanism induced by sand fly saliva against Leishmania infection. Sensitization by uninfected sand fly bites (1) or immunization by injecting a single sand fly salivary molecule (2) leads to uptake of salivary protein(s) by antigen presenting cells (APCs) that traffic to the lymph nodes where they prime naïve $C D 4^{+} \mathrm{T}$ cells (3). When an immune host is challenged by a Leishmania-infected sand fly bite (4) it triggers a rapid recruitment of anti-saliva memory T cells, mostly $\mathrm{CD}^{+}$producing IFN- $\gamma$, from the draining lymph nodes (dashed arrows) to the site of the sand fly bite. Cellular recruitment generally peaks at $48 \mathrm{~h}$ after challenge by bite characterized as a delayed-type hypersensitivity reaction (DTH) (5). CD4 ${ }^{+}$T cells producing IFN- $\gamma$ will activate macrophages (M $\left.\phi\right)$ promoting Leishmania killing (Th1 response) (6). another plasmid coding for a different DTH-inducing salivary protein from P. papatasi, PpSP44, resulted in disease enhancement after $L$. major infection, suggesting that distinct molecules from the same vector can generate different immune responses leading to opposite outcomes of parasite infection (Oliveira et al., 2008). Although PpSP44 was able to induce a cellular recruitment after recall by parasite plus saliva or by sand fly bites, the cells did not produce IFN- $\gamma$, but did produce IL-4. This result suggests that an immune response specific to a salivary antigen that induces a cellular immune response (DTH) and IFN- $\gamma$ production is able to confer protection against L. major infection. This hypothesis has been validated in another model with a salivary protein from $L$. longipalpis, LJM19, tested in a hamster model of VL (Gomes et al., 2008). Hamsters immunized with LJM19 and challenged with $L$. infantum plus L. longipalpis SGH, were able to maintain a low parasite load in the spleen and liver 5 months after infection, with an overall high IFN- $\gamma /$ TGF- $\beta$ ratio and inducible NOS expression in both organs. LJM19-immunized hamsters induced a strong DTH with IFN- $\gamma$ production $48 \mathrm{~h}$ after exposure to uninfected sand fly bites, a consistent predictor of protection. The expression of IFN$\gamma$ at the site of bite can partly explain the reduced number of $L$. infantum in the spleen and liver of LJM19-immunized hamsters. 
The early immune response generated by LJM19 could promote a direct parasite killing and/or priming of anti-Leishmania immunity. In the same study, another salivary protein of L. longipalpis, LJM11, protected hamsters against VL up to 2 months postinfection with L. infantum (Gomes et al., 2008). The protection was partial and associated with a DTH response composed mainly of macrophages and lymphocytes. More recently, when tested in a model of CL, LJM11 conferred protective immunity against $L$. major infection (Xu et al., 2011). The protection was correlated with a strong DTH induced in C57BL/6 strain mouse following exposure to L. longipalpis SGH. Additionally, splenocytes of mice exposed to uninfected sand fly bites produced IFN- $\gamma$ upon stimulation with recombinant LJM11 protein, suggesting the induction of a systemic Th1 immunity by this molecule. Using the hamster model of VL, da Silva et al. (2011) combined LJM19 with KMP11, a DNA plasmid coding Leishmania antigen, and showed a protective effect in hamsters after challenge with $L$. infantum plus L. longipalpis SGH. The combined plasmids induced IFN- $\gamma$ production in the draining lymph nodes at 7, 14, and 21 days post-immunization. Increased IFN- $\gamma /$ IL- 10 and IFN- $\gamma /$ TGF- $\beta$ ratios were observed in the spleen 2 and 5 months after challenge with a subsequent reduction in parasite load in the spleen and liver. Like LJM11, LJM19 also showed the capacity for inducing a protective immune response against different species of Leishmania transmitted by different species of sand fly. Hamsters immunized with LJM19 controlled the infection against $L$. braziliensis plus SGH of $L$. intermedia, its natural vector (Tavares et al., 2011). Table 2 summarizes the role of sand fly salivary proteins in protection against Leishmania infection. The ability of these salivary proteins to induce Th1 cytokines is remarkable, considering the absence of adjuvants. It remains unknown how these sand fly salivary proteins efficiently stimulate the immune system triggering a Th1 response. We believe that these protective salivary proteins may be interacting with the innate immune system, working in a self-adjuvant manner. Taken together, an early anti-saliva cellular immunity (DTH) and the presence of a Th1 immune response mainly characterized by the presence of IFN- $\gamma$ against the salivary proteins inoculated during challenge, alter the outcome of Leishmania infection and correlates with protection. It reinforces the concept of using components of arthropod saliva as a novel strategy against vector-borne diseases.

\section{CHALLENGES FOR VACCINE DEVELOPMENT}

The immune response to sand fly salivary proteins has been consistently shown to protect rodents against leishmaniasis in laboratory settings. From identification in rodents to translation into marketable vaccine candidates, protective sand fly salivary proteins have to overcome several hurdles including intrinsic genetic variation among sand fly populations, variations between field and colonized sand flies, and the possibility of causing allergies or desensitization.

Recently, researchers working with $P$. papatasi in Tunisia (Ahmed et al., 2010; Ben Hadj Ahmed et al., 2010, 2011) showed that colonization of sand flies favors protection against Leishmania. Protection accomplished using long-term colonized sand flies (F29) could not be reproduced with first generation $P$. papatasi. Surprisingly, colonization for five generations led to the acquisition of the protective qualities against Leishmaniasis of sand fly saliva. The nature of the changes remains unknown. One possible explanation for these findings may be related to amount of salivary protein present in the colonized vs. wild caught flies rather than a change or a rapid loss of genetic variability. It is very difficult to estimate the amount of salivary proteins in wild caught flies compared to colonized flies in which the maximum amount will normally peak after day 4 and will decrease after day 12. Therefore, these experiments may need to be repeated normalizing the amount of salivary proteins when using colonized vs. wild caught flies. In fact, Laurenti et al. (2009a,b) showed that salivary proteins from field-captured $L$. longipalpis impacted the immune system differently from laboratory-reared sand flies. Nevertheless, this work clearly indicates (by SDS-PAGE analysis) that one salivary gland pair from wild caught sand flies had fewer amounts of proteins when compared to a salivary gland pair from colonized flies. This supports the likelihood that amount and not the type of saliva is responsible for these effects. Currently, most of the published work on sand fly saliva immunity relies on longterm colonized sand flies. We have compared by transcriptomic

Table 2 | Sand fly salivary proteins tested in animal model of leishmaniasis.

\begin{tabular}{|c|c|c|c|c|}
\hline Salivary protein candidate (sand fly) & Challenged with & Immune response & Protection & Reference \\
\hline $\operatorname{PpSP} 15^{2}$ (P. papatasi) ${ }^{4}$ & L. major + P. papatasi SGH & Cellular and humoral & Yes & Valenzuela et al. (2001) \\
\hline LJM19² (L. longipalpis) ${ }^{3}$ & L. infantum + L. longipalpis SGH & Cellular & Yes & Gomes et al. (2008) \\
\hline LJM19² (L. longipalpis) ${ }^{3}$ & L. infantum + L. longipalpis SGH & Cellular & Yes & da Silva et al. (2011) \\
\hline LJM17² (L. longipalpis) ${ }^{3}$ & L. infantum + L. longipalpis SGH & Cellular and humoral & No & Gomes et al. (2008) \\
\hline LJL112 (L. longipalpis) ${ }^{3}$ & L. infantum + L. longipalpis SGH & Humoral & No & Gomes et al. (2008) \\
\hline LJM112 (L. longipalpis) ${ }^{3}$ & L. infantum + L. longipalpis SGH & Cellular and humoral & Partial & Gomes et al. (2008) \\
\hline LJM11² (L. longipalpis) ${ }^{4}$ & L. major + L. longipalpis SGH & Cellular and humoral & Yes & Xu et al. (2011) \\
\hline LJL143² (L. longipalpis) ${ }^{4}$ & L. major + L. longipalpis SGH & Cellular & No & Xu et al. (2011) \\
\hline
\end{tabular}

${ }^{1}$ Peptide immunization; ${ }^{2}$ DNA immunization; ${ }^{3} \mathrm{VL}$ model; ${ }^{4} \mathrm{CL}$ model. 
analysis colonized vs. wild caught flies even from different geographical areas and to our surprise the level of identity between the different salivary transcripts was very high (Kato et al., 2006). However, it will be important, to perform an in depth proteomics or deep sequencing analysis to determine if there is a specific difference between the salivary proteins of colonized and wild caught sand flies.

Individuals from endemic areas are likely bitten by sand flies everyday and possibly many times a day. The effect of multiple exposures has been investigated in $\mathrm{BALB} / \mathrm{c}$ mice that were exposed to bites of thirty $P$. duboscqi females every week for 15 weeks (Rohousova et al., 2011). After multiple exposures the mice lost the ability to mount a protective anti-saliva immune response. This raised concerns that humans living in endemic areas may be desensitized over time. This hypothesis needs to be tested in humans from areas where sand flies are prevalent.

Another possible obstacle that needs to be addressed is the genetic variation among populations of sand flies. It has been reported that some salivary proteins, i.e., maxadilan from $L$. longipalpis, are highly polymorphic depending on geographical location (Lanzaro et al., 1999). Conversely, PpSP15 from $P$. papatasi has been shown to have little diversifying selection being mostly conserved at the amino acid level from different populations in Sudan, Egypt, Jordan, Israel, and Saudi Arabia (Elnaiem et al., 2005). These data indicate that a vaccine candidate from sand fly salivary proteins that is conserved and would potentially work across different geographical locations can be identified.

We also advocate that vaccine candidates to leishmaniasis need to be tested against a Leishmania-infected sand fly-challenge. Peters et al. (2009) have demonstrated that a challenge with

\section{REFERENCES}

Abdeladhim, M., Ben Ahmed, M., Marzouki, S., Belhadj Hmida, N., Boussoffara, T., Belhaj Hamida, N., Ben Salah, A., and Louzir, H. (2011). Human cellular immune response to the saliva of Phlebotomus papatasi is mediated by IL-10-producing CD8+ $\mathrm{T}$ cells and Th1-polarized CD4+ lymphocytes. PLoS Negl. Trop. Dis. 5, e1345. doi:10.1371/journal.pntd.0001345

Ahmed, S. B., Kaabi, B., Chelbi, I., Derbali, M., Cherni, S., Laouini, D., and Zhioua, E. (2010). Lack of protection of pre-immunization with saliva of long-term colonized Phlebotomus papatasi against experimental challenge with Leishmania major and saliva of wild-caught $P$. papatasi. Am. J. Trop. Med. Hyg. 83, 512-514.

Aquino, D. M., Caldas, A. J., Miranda, J. C., Silva, A. A., Barral-Netto, M., and Barral, A. (2010). Epidemiological study of the association between anti-Lutzomyia longipalpis saliva antibodies and development of delayed-type hypersensitivity to

infected sand flies is more virulent, overcoming the protection otherwise conferred by a Leishmania vaccine to the challenge with parasites by needle inoculation. The infected challenge incorporates several unique features: 1-sand fly saliva; 2 -promatigote secretory gel (Rogers et al., 2004, 2009); 3-infective metacyclic Leishmania parasites; 4-Sand fly probing; and damage of the host's skin during the transmission. This is of great significance and complexity and will be difficult to mimic. Therefore, vector challenge should be used in order to advance vaccine development toward an effective Leishmania vaccine for humans.

\section{CONCLUSION}

Immunity against sand fly salivary proteins and their potential as components of a Leishmania vaccine continues to be overlooked. While significant progress has been made to understand the immune response to sand fly saliva in humans and rodents, a deeper understanding of the contributions of the innate and adaptive immune system is needed. Studies in rodents where sand fly salivary proteins are tested in conjunction with parasite candidates are desirable. Moreover, more studies in humans and canids from endemic areas correlating anti-saliva cellular immune response and leishmaniasis outcome are essential for the advancement of the vector-Leishmania field. The experimental studies reviewed here highlight that immunity to salivary proteins are a promising supplement to conventional anti-Leishmania vaccine approaches.

\section{ACKNOWLEDGMENTS}

We thank Dr. Jesus Valenzuela, Dr. Shaden Kamhawi, and Dana C. Gilmore for critical revision of this manuscript. The National Institutes of Health, NIAID, and Intramural Research Program supported this work.

epidemiological marker? Am. J. Trop. Med. Hyg. 62, 740-745.

Belkaid, Y., Kamhawi, S., Modi, G., Valenzuela, J., Noben-Trauth, N., Rowton, E., Ribeiro, J., and Sacks, D. L. (1998). Development of a natural model of cutaneous leishmaniasis: powerful effects of vector saliva and saliva preexposure on the long-term outcome of Leishmania major infection in the mouse ear dermis. J. Exp. Med. 188, 1941-1953.

Belkaid, Y., Valenzuela, J. G., Kamhawi, S., Rowton, E., Sacks, D. L., and Ribeiro, J. M. (2000). Delayedtype hypersensitivity to Phlebotomus papatasi sand fly bite: an adaptive response induced by the fly? Proc. Natl. Acad. Sci. U.S.A. 97, 6704-6709.

Ben Hadj Ahmed, S., Chelbi, I., Kaabi, B., Cherni, S., Derbali, M., and Zhioua, E. (2010). Differences in the salivary effects of wild-caught versus colonized Phlebotomus papatasi (Diptera: Psychodidae) on the development of zoonotic cutaneous leishmaniasis in $\mathrm{BALB} / \mathrm{c}$ mice. J. Med. Entomol. 47, 74-79.
Ben Hadj Ahmed, S., Kaabi, B., Chelbi, I., Cherni, S., Derbali, M., Laouini, D., and Zhioua, E. (2011). Colonization of Phlebotomus papatasi changes the effect of pre-immunization with saliva from lack of protection towards protection against experimental challenge with Leishmania major and saliva. Parasit. Vectors 4, 126.

Brodie, T. M., Smith, M. C., Morris, R. V., and Titus, R. G. (2007). Immunomodulatory effects of the Lutzomyia longipalpis salivary gland protein maxadilan on mouse macrophages. Infect. Immun. 75, 2359-2365.

Carregaro, V., Sa-Nunes, A., Cunha, T. M., Grespan, R., Oliveira, C. J., LimaJunior, D. S., Costa, D. L., Verri, W. A. Jr., Milanezi, C. M., Pham, V. M., Brand, D. D., Valenzuela, J. G., Silva, J. S., Ribeiro, J. M., and Cunha, F. Q. (2011). Nucleosides from Phlebotomus papatasi salivary gland ameliorate murine collageninduced arthritis by impairing dendritic cell functions. J. Immunol. 187, 4347-4359. 
Charlab, R., Valenzuela, J. G., Rowton, E. D., and Ribeiro, J. M. (1999). Toward an understanding of the biochemical and pharmacological complexity of the saliva of a hematophagous sand fly Lutzomyia longipalpis. Proc. Natl. Acad. Sci. U.S.A. 96, 15155-15160.

Clements, M. F., Gidwani, K., Kumar, R., Hostomska, J., Dinesh, D. S., Kumar, V., Das, P., Muller, I., Hamilton, G., Volfova, V., Boelaert, M., Das, M., Rijal, S., Picado, A., Volf, P., Sundar, S., Davies, C. R., and Rogers, M. E. (2010). Measurement of recent exposure to Phlebotomus argentipes, the vector of Indian visceral Leishmaniasis, by using human antibody responses to sand fly saliva. Am. J. Trop. Med. Hyg. 82, 801-807.

Collin, N., Gomes, R., Teixeira, C., Cheng, L., Laughinghouse, A., Ward, J. M., Elnaiem, D. E., Fischer, L., Valenzuela, J. G., and Kamhawi, S. (2009). Sand fly salivary proteins induce strong cellular immunity in a natural reservoir of visceral leishmaniasis with adverse consequences for Leishmania. PLoS Pathog. 5, e1000441. doi:10.1371/journal.ppat.1000441

Costa, D. J., Favali, C., Clarencio, J., Afonso, L., Conceicao, V., Miranda, J. C., Titus, R. G., Valenzuela, J., BarralNetto, M., Barral, A., and Brodskyn, C. I. (2004). Lutzomyia longipalpis salivary gland homogenate impairs cytokine production and costimulatory molecule expression on human monocytes and dendritic cells. Infect. Immun. 72, 1298-1305.

da Silva, R. A., Tavares, N. M., Costa, D., Pitombo, M., Barbosa, L., Fukutani, K., Miranda, J. C., De Oliveira, C. I., Valenzuela, J. G., Barral, A., Soto, M., Barral-Netto, M., and Brodskyn, C. (2011). DNA vaccination with KMP11 and Lutzomyia longipalpis salivary protein protects hamsters against visceral leishmaniasis. Acta Trop. 120, 185-190.

de Moura, T. R., Oliveira, F., Novais, F. O., Miranda, J. C., Clarencio, J., Follador, I., Carvalho, E. M., Valenzuela, J. G., Barral-Netto, M., Barral, A., Brodskyn, C., and De Oliveira, C. I. (2007). Enhanced Leishmania braziliensis infection following preexposure to sandfly saliva. PLoS Negl. Trop. Dis. 1, e84. doi:10.1371/journal.pntd.0000084

Desjeux, P. (2004). Leishmaniasis. Nat. Rev. Microbiol. 2, 692.

Drahota, J., Lipoldova, M., Volf, P., and Rohousova, I. (2009). Specificity of anti-saliva immune response in mice repeatedly bitten by Phlebotomus sergenti. Parasite Immunol. 31, 766-770.
Elnaiem, D. E., Meneses, C., Slotman, M., and Lanzaro, G. C. (2005). Genetic variation in the sand fly salivary protein, SP-15, a potential vaccine candidate against Leishmania major. Insect Mol. Biol. 14, 145-150.

Ghosh, K. N., and Mukhopadhyay, J. (1998). The effect of anti-sandfly saliva antibodies on Phlebotomus argentipes and Leishmania donovani. Int. J. Parasitol. 28, 275-281.

Gillespie, R. D., Mbow, M. L., and Titus, R. G. (2000). The immunomodulatory factors of blood feeding arthropod saliva. Parasite Immunol. 22, 319-331.

Gomes, R., Teixeira, C., Teixeira, M. J., Oliveira, F., Menezes, M. J., Silva, C., De Oliveira, C. I., Miranda, J. C., Elnaiem, D. E., Kamhawi, S., Valenzuela, J. G., and Brodskyn, C. I. (2008). Immunity to a salivary protein of a sand fly vector protects against the fatal outcome of visceral leishmaniasis in a hamster model. Proc. Natl. Acad. Sci. U.S.A. 105, 7845-7850.

Gomes, R. B., Brodskyn, C., De Oliveira, C. I., Costa, J., Miranda, J. C., Caldas, A., Valenzuela, J. G., BarralNetto, M., and Barral, A. (2002). Seroconversion against Lutzomyia longipalpis saliva concurrent with the development of anti-Leishmania chagasi delayed-type hypersensitivity. J. Infect. Dis. 186, 1530-1534

Gomes, R. B., Mendonca, I. L., Silva, V. C., Ruas, J., Silva, M. B., Cruz, M. S., Barral, A., and Costa, C. H. (2007). Antibodies against Lutzomyia longipalpis saliva in the fox Cerdocyon thous and the sylvatic cycle of Leishmania chagasi. Trans. R. Soc. Trop. Med. Hyg. 101, 127-133.

Hasko, G., Kuhel, D. G., Chen, J. F., Schwarzschild, M. A., Deitch, E. A., Mabley, J. G., Marton, A., and Szabo, C. (2000). Adenosine inhibits IL-12 and TNF-[alpha] production via adenosine A2a receptordependent and independent mechanisms. FASEB J. 14, 2065-2074.

Hasko, G., Szabo, C., Nemeth, Z. H., Kvetan, V., Pastores, S. M., and Vizi, E. S. (1996). Adenosine receptor agonists differentially regulate IL-10, TNF-alpha, and nitric oxide production in RAW 264.7 macrophages and in endotoxemic mice. J. Immunol. 157, 4634-4640.

Hostomska, J., Rohousova, I., Volfova, V., Stanneck, D., Mencke, N., and Volf, P. (2008). Kinetics of canine antibody response to saliva of the sand fly Lutzomyia longipalpis. Vector Borne Zoonotic Dis. 8, 443-450.

Jochim, R. C., Teixeira, C. R., Laughinghouse, A., Mu, J., Oliveira, F.,
Gomes, R. B., Elnaiem, D. E., and Valenzuela, J. G. (2008). The midgut transcriptome of Lutzomyia longipalpis: comparative analysis of cDNA libraries from sugar-fed, blood-fed, post-digested and Leishmania infantum chagasi-infected sand flies. BMC Genomics 9, 15 doi:10.1186/1471-2164-9-15

Kamhawi, S. (2000). The biological and immunomodulatory properties of sand fly saliva and its role in the establishment of Leishmania infections. Microbes Infect. 2, 1765-1773.

Kamhawi, S., Belkaid, Y., Modi, G. Rowton, E., and Sacks, D. (2000). Protection against cutaneous leishmaniasis resulting from bites of uninfected sand flies. Science 290, 1351-1354.

Kato, H., Anderson, J. M., Kamhawi, S., Oliveira, F., Lawyer, P. G., Pham, V. M., Sangare, C. S., Samake, S., Sissoko, I., Garfield, M., Sigutova, L., Volf, P., Doumbia, S., and Valenzuela, J. G. (2006). High degree of conservancy among secreted salivary gland proteins from two geographically distant Phlebotomus duboscq sandflies populations (Mali and Kenya). BMC Genomics 7, 226. doi:10.1186/1471-2164-7-22

Kato, H., Jochim, R. C., Lawyer, P. G., and Valenzuela, J. G. (2007). Identification and characterization of a salivary adenosine deaminase from the sand fly Phlebotomus duboscqi, the vector of Leishmania major in sub-Saharan Africa. J. Exp. Biol. 210, 733-740.

Katz, O., Waitumbi, J. N., Zer, R., and Warburg, A. (2000). Adenosine, AMP, and protein phosphatase activity in sandfly saliva. Am. J. Trop. Med. Hyg. 62, 145-150.

Lanzaro, G. C., Lopes, A. H., Ribeiro, J. M., Shoemaker, C. B., Warburg, A., Soares, M., and Titus, R. G. (1999). Variation in the salivary peptide, maxadilan, from species in the Lutzomyia longipalpis complex. Insect Mol. Biol. 8, 267-275.

Laurenti, M. D., Da Matta, V. L., Pernichelli, T., Secundino, N. F., Pinto, L. C., Corbett, C. E., and Pimenta, P. P. (2009a). Effects of salivary gland homogenate from wildcaught and laboratory-reared Lutzomyia longipalpis on the evolution and immunomodulation of Leishmania (Leishmania) amazonensis infection. Scand. J. Immunol. 70, 389-395.

Laurenti, M. D., Silveira, V. M., Secundino, N. F., Corbett, C. E., and Pimenta, P. P. (2009b). Saliva of laboratory-reared Lutzomyia longipalpis exacerbates Leishmania
(Leishmania) amazonensis infection more potently than saliva of wild-caught Lutzomyia longipalpis. Parasitol. Int. 58, 220-226.

Lerner, E. A., Ribeiro, J. M., Nelson, R. J., and Lerner, M. R. (1991). Isolation of maxadilan, a potent vasodilatory peptide from the salivary glands of the sand fly Lutzomyia longipalpis. J. Biol. Chem. 266, 11234-11236.

Marzouki, S., Ben Ahmed, M., Boussoffara, T., Abdeladhim, M., Ben AleyaBouafif, N., Namane, A., Hamida, N. B., Ben Salah, A., and Louzir, H. (2011). Characterization of the antibody response to the saliva of Phlebotomus papatasi in people living in endemic areas of cutaneous leishmaniasis. Am. J. Trop. Med. Hyg. 84, 653-661.

Menezes, M. J., Costa, D. J., Clarencio, J., Miranda, J. C., Barral, A., BarralNetto, M., Brodskyn, C., and De Oliveira, C. I. (2008). Immunomodulation of human monocytes following exposure to Lutzomyia intermedia saliva. BMC Immunol. 9, 12. doi:10.1186/1471-2172-9-12

Morris, R. V., Shoemaker, C. B., David, J. R., Lanzaro, G. C., and Titus, R. G. (2001). Sandfly maxadilan exacerbates infection with Leishmania major and vaccinating against it protects against L. major infection. J. Immunol. 167, 5226-5230.

Oliveira, F., Jochim, R. C., Valenzuela, J. G., and Kamhawi, S. (2009). Sand flies, Leishmania, and transcriptome-borne solutions. Parasitol. Int. 58, 1-5.

Oliveira, F., Kamhawi, S., Seitz, A. E., Pham, V. M., Guigal, P. M. Fischer, L., Ward, J., and Valenzuela, J. G. (2006). From transcriptome to immunome: identification of DTH inducing proteins from a Phlebotomus ariasi salivary gland cDNA library. Vaccine 24, 374-390.

Oliveira, F., Lawyer, P. G., Kamhawi, S., and Valenzuela, J. G. (2008). Immunity to distinct sand fly salivary proteins primes the anti-Leishmania immune response towards protection or exacerbation of disease. PLoS Negl. Trop. Dis. 2, e226. doi:10.1371/journal.pntd.0000226

Peters, N. C., Kimblin, N., Secundino, N., Kamhawi, S., Lawyer, P., and Sacks, D. L. (2009). Vector transmission of Leishmania abrogates vaccine-induced protective immunity. PLoS Pathog. 5, e1000484. doi:10.1371/journal.ppat.1000484

Prates, D. B., Araujo-Santos, T., Luz, N. F., Andrade, B. B., Franca-Costa, J., Afonso, L., Clarencio, J., Miranda, J. 
C., Bozza, P. T., Dosreis, G. A., Brodskyn, C., Barral-Netto, M., Borges, V. M., and Barral, A. (2011). Lutzomyia longipalpis saliva drives apoptosis and enhances parasite burden in neutrophils. J. Leukoc. Biol. 90, 575-582.

Qureshi, A. A., Asahina, A., Ohnuma, M., Tajima, M., Granstein, R. D., and Lerner, E. A. (1996). Immunomodulatory properties of maxadilan, the vasodilator peptide from sand fly salivary gland extracts. Am. J. Trop. Med. Hyg. 54, 665-671.

Ribeiro, J. M. (1987). Role of saliva in blood-feeding by arthropods. Annu. Rev. Entomol. 32, 463-478.

Ribeiro, J. M., Katz, O., Pannell, L. K., Waitumbi, J., and Warburg, A. (1999). Salivary glands of the sand fly Phlebotomus papatasi contain pharmacologically active amounts of adenosine and $5^{\prime}$-AMP. J. Exp. Biol. 202, 1551-1559.

Rogers, M., Kropf, P., Choi, B. S., Dillon, R., Podinovskaia, M., Bates, P., and Muller, I. (2009). Proteophosophoglycans regurgitated by Leishmania-infected sand flies target the L-arginine metabolism of host macrophages to promote parasite survival. PLoS Pathog. 5, e1000555. doi:10.1371/journal.ppat. 1000555

Rogers, M. E., Ilg, T., Nikolaev, A. V., Ferguson, M. A., and Bates, P. A. (2004). Transmission of cutaneous leishmaniasis by sand flies is enhanced by regurgitation of fPPG. Nature 430, 463-467.

Rohousova, I., Hostomska, J., Vlkova, M., Kobets, T., Lipoldova, M., and Volf, P. (2011). The protective effect against Leishmania infection conferred by sand fly bites is limited to short-term exposure. Int. J. Parasitol. 41, 481-485.

Rohousova, I., Ozensoy, S., Ozbel, Y., and Volf, P. (2005a). Detection of species-specific antibody response of humans and mice bitten by sand flies. Parasitology 130, 493-499.
Rohousova, I., Volf, P., and Lipoldova, M. (2005b). Modulation of murine cellular immune response and cytokine production by salivary gland lysate of three sand fly species. Parasite Immunol. 27, 469-473.

Silva, F., Gomes, R., Prates, D., Miranda, J. C., Andrade, B., Barral-Netto, M., and Barral, A. (2005). Inflammatory cell infiltration and high antibody production in $\mathrm{BALB} / \mathrm{c}$ mice caused by natural exposure to Lutzomyia longipalpis bites. Am. J. Trop. Med. Hyg. 72, 94-98.

Soares, M. B., Titus, R. G., Shoemaker, C. B., David, J. R., and Bozza, M. (1998). The vasoactive peptide maxadilan from sand fly saliva inhibits TNF-alpha and induces IL6 by mouse macrophages through interaction with the pituitary adenylate cyclase-activating polypeptide (PACAP) receptor. J. Immunol. 160, 1811-1816

Souza, A. P., Andrade, B. B., Aquino, D., Entringer, P., Miranda, J. C., Alcantara, R., Ruiz, D., Soto, M., Teixeira, C. R., Valenzuela, J. G., De Oliveira, C. I., Brodskyn, C. I., Barral-Netto, M., and Barral, A. (2010). Using recombinant proteins from Lutzomyia longipalpis saliva to estimate human vector exposure in visceral Leishmaniasis endemic areas. PLoS Negl. Trop. Dis. 4, e649. doi:10.1371/journal.pntd.0000649

Tavares, N. M., Silva, R. A., Costa, D. J., Pitombo, M. A., Fukutani, K. F., Miranda, J. C., Valenzuela, J. G., Barral, A., De Oliveira, C. I., BarralNetto, M., and Brodskyn, C. (2011). Lutzomyia longipalpis saliva or salivary protein LJM19 protects against Leishmania braziliensis and the saliva of its vector, Lutzomyia intermedia. PLoS Negl. Trop. Dis. 5, el169. doi:10.1371/journal.pntd.0001169

Teixeira, C., Gomes, R., Collin, N., Reynoso, D., Jochim, R., Oliveira, F., Seitz, A., Elnaiem, D. E., Caldas, A., De Souza, A. P., Brodskyn, C. I., De
Oliveira, C. I., Mendonca, I., Costa, C. H., Volf, P., Barral, A., Kamhawi, S., and Valenzuela, J. G. (2010). Discovery of markers of exposure specific to bites of Lutzomyia longipalpis, the vector of Leishmania infantum chagasi in Latin America. PLoS Negl. Trop. Dis. 4, e638. doi:10.1371/journal.pntd.0000638

Thiakaki, M., Rohousova, I., Volfova, V., Volf, P., Chang, K. P., and Soteriadou, K. (2005). Sand fly specificity of saliva-mediated protective immunity in Leishmania amazonensis-BALB/c mouse model. Microbes Infect. 7, 760-766.

Titus, R. G., and Ribeiro, J. M. (1988). Salivary gland lysates from the sand fly Lutzomyia longipalpis enhance Leishmania infectivity. Science 239 1306-1308.

Valenzuela, J. G., Belkaid, Y., Garfield, M. K., Mendez, S., Kamhawi, S., Rowton, E. D., Sacks, D. L., and Ribeiro, J. M. (2001). Toward a defined antiLeishmania vaccine targeting vector antigens: characterization of a protective salivary protein. J. Exp. Med. 194, 331-342.

Vinhas, V., Andrade, B. B., Paes, F. Bomura, A., Clarencio, J., Miranda, J. C., Bafica, A., Barral, A., and Barral-Netto, M. (2007). Human anti-saliva immune response following experimental exposure to the visceral leishmaniasis vector, Lutzomyia longipalpis. Eur. J. Immunol. 37, 3111-3121.

Vlkova, M., Rohousova, I., Drahota, J. Stanneck, D., Kruedewagen, E. M., Mencke, N., Otranto, D., and Volf, P. (2011). Canine antibody response to Phlebotomus perniciosus bites negatively correlates with the risk of Leishmania infantum transmission. PLoS Negl. Trop. Dis. 5, e1344. doi:10.1371/journal.pntd.0001344

Wheat, W. H., Pauken, K. E., Morris, R. V., and Titus, R. G. (2008). Lutzomyia longipalpis salivary peptide maxadilan alters murine dendritic cell expression of CD80/86, CCR7, and cytokine secretion and reprograms dendritic cell-mediated cytokine release from cultures containing allogeneic $\mathrm{T}$ cells. J. Immunol. 180, 8286-8298.

WHO. (2010). Control of the Leishmaniasis: Report of a Meeting of the WHO Expert Committee on the Control of Leishmaniases. WHO Technical Report Series, No 949. Geneva: WHO.

Xu, X., Oliveira, F., Chang, B. W., Collin, N., Gomes, R., Teixeira, C., Reynoso, D., Pham, V. M., Elnaiem, D.-E. Kamhawi, S., Ribeiro, J. M. C., Valenzuela, J. G., and Andersen, J. F. (2011). Structure and function of a "yellow" protein from saliva of the sand fly Lutzomyia longipalpis that confers protective immunity against Leishmania major infection. J. Biol. Chem. 286, 32383-32393.

Conflict of Interest Statement: The authors declare that the research was conducted in the absence of any commercial or financial relationships that could be construed as a potential conflict of interest.

Received: 11 January 2012; accepted: 19 April 2012; published online: 11 May 2012.

Citation: Gomes R and Oliveira F (2012) The immune response to sand fly salivary proteins and its influence on Leishmania immunity. Front. Immun. 3:110. doi: 10.3389/fimmu.2012.00110

This article was submitted to Frontiers in Microbial Immunology, a specialty of Frontiers in Immunology.

Copyright (C) 2012 Gomes and Oliveira. This is an open-access article distributed under the terms of the Creative Commons Attribution Non Commercial License, which permits non-commercial use, distribution, and reproduction in other forums, provided the original authors and source are credited. 